\title{
Reflets
}

Revue ontaroise d'intervention sociale et communautaire

\section{La communication : le pont entre les Sourds et les entendants}

\section{Ann Toth}

Volume 4, numéro 2, automne 1998

Personnes vivant avec une incapacité

URI : https://id.erudit.org/iderudit/026221ar

DOI : https://doi.org/10.7202/026221ar

Aller au sommaire du numéro

Éditeur(s)

Reflets : Revue ontaroise d'intervention sociale et communautaire

ISSN

1203-4576 (imprimé)

1712-8498 (numérique)

Découvrir la revue

Citer cet article

Toth, A. (1998). La communication : le pont entre les Sourds et les entendants. Reflets, 4(2), 115-122. https://doi.org/10.7202/026221ar

Tous droits réservés (C) Reflets : Revue ontaroise d'intervention sociale et communautaire, 1998
Ce document est protégé par la loi sur le droit d'auteur. L'utilisation des services d'Érudit (y compris la reproduction) est assujettie à sa politique d'utilisation que vous pouvez consulter en ligne.

https://apropos.erudit.org/fr/usagers/politique-dutilisation/ 


\title{
La communication : le pont entre les Sourds et les entendants
}

\author{
Ann Toth \\ Travailleuse sociale, Sarnia
}

La pratique du travail social auprès des personnes sourdes comporte de nombreux défis. En classant les Sourds comme handicapés, on se refuse à voir les différences linguistiques et culturelles et on nie du même coup que les personnes sourdes puissent être sur le même pied d'égalité que celles qui cherchent à remédier à leurs difficultés. Les travailleurs sociaux, parce qu'ils ont reçu une formation leur permettant d'envisager les problèmes du point de vue de leurs clients, possèdent cette aptitude à reconnaitre ces différences et à tabler sur elles pour aider leurs clients à s'aider eux-mêmes. Le présent article propose un modèle qui respecte les différences linguistiques et culturelles entre les Sourds et les entendants, tout en promouvant la communication saine et la résolution de problèmes.

La documentation relative aux aspects linguistiques et culturels de la surdité souligne que les termes «sourd» et «Sourd» comportent des connotations différentes. La première variante reflète une limitation physique, tandis que la seconde renvoie à des caractéristiques linguistiques et culturelles qui impliquent une toute autre dimension, voire une toute autre identité. La première variante est particulièrement visible dans les critères d'admission aux écoles pour les personnes sourdes, où les enfants ayant une perte auditive déterminée sont considérés comme "sourds», et dans la Loi canadienne sur l'Éducation de 1990, où les «sourds» ont droit à des appareils fonctionnels de même qu'à une éducation spéciale. 
En revanche, le mot «Sourd» évoque une identité linguistique et culturelle différente. Qu'ils satisfassent ou non aux exigences de déficience auditive, les Sourds utilisent le langage gestuel (LSQ, langue des signes québécoise ou ASL, langue des signes américaine) et sont aussi reconnus comme membres d'un groupe culturel (par exemple, la famille, les amis entendants ou malentendants des Sourds) (Woodward 1972).

Selon Watzlawick, Beavin et Jackson (1967), on ne peut s'empêcher de communiquer. Notre nature même nous pousse, dès la conception, à nous exprimer, à nous tailler une place dans le monde et à créer des rapports avec ceux qui nous entourent. Ce besoin d'établir un lien avec le monde nous incite à faire appel à la communication, peu importe sa forme, pour assurer notre survie. La seule pensée que nos efforts porteront fruit, écarte la peur de la séparation et nous rassure sur notre existence (Bowlby 1973). Même avant qu'une langue formelle ne soit comprise ou exprimée, la façon dont nos parents nous bercent et nous aiment parle plus fort que les mots.

$\mathrm{Au}$ fur et à mesure que notre système biologique de communication répond à notre environnement, l'acquisition et la maîtrise de la langue deviennent des tâches de plus en plus importantes (Bowlby 1973). Surgit alors une question importante : quelle est la solution qui me convient le mieux? Compte tenu du choix entre le langage parlé et le langage gestuel, c'est l'expérimentation qui, en définitive, nous conduira au mode de communication correspondant le mieux à nos besoins.

Sans que nous nous en rendions compte, le cerveau choisit ce qui nous convient le mieux: nos approximations orales ou gestuelles. Avant même de subir une quelconque évaluation audiologique, nous voilà placés devant une alternative qui nous définira, non pas en tant qu'entendants ou personnes sourdes, mais en tant qu'êtres humains pourvus d'une identité propre qui s'appuie sur les moyens utilisés pour communiquer avec les autres. Cela dit, nos tuteurs (parents, professeurs, etc.) renforceront nos efforts de façon à ce que notre mode de communication cadre avec leurs méthodes préférées (Pettito et Marentette 1991). 
Plusieurs études signalent que, peu importe le type de communication adopté, les enfants sourds acquièrent des automatismes langagiers à la même vitesse que les enfants entendants (Pettito et Marentette 1991). Ainsi, que le mode de communication soit visuel ou gestuel chez l'enfant sourd ou élevé dans une famille de personnes sourdes ou encore, oral chez l'enfant entendant élevé au sein d'une famille d'entendants, les enfants établiront spontanément un système de communication. On a aussi découvert qu'à l'exception des enfants victimes de violence physique ou psychologique, cette capacité de participer à une conversation est, en dépit de leur éventuelle incapacité, un don commun à tous les enfants.

Tel que l'a souligné Fascold (1984), si nous utilisons la langue pour communiquer, nous nous en servons également pour évaluer l'aspect social de notre environnement. La langue permet d'exprimer notre identité sociale et culturelle, d'affirmer notre appartenance à certains groupes, de traduire les rapports que nous avons avec les autres et de décrire la situation vécue. Ainsi, la langue est à la fois outil de communication et outil social (Lucas, 1989).

Ceci dit, il importe de relever les raisons pour lesquelles les entendants et les Sourds utilisent un mode de communication différent. Évidemment, les personnes sourdes et les entendants se distinguent par leurs différences biologiques, en ce qui touche la façon dont la langue est assimilée et transmise. Mais les différences résident également dans le choix entre le langage oral, visuel ou écrit ou encore dans l'utilisation d'une combinaison de ces trois formes de communication.

La préférence qu'on accorde à une langue dominante, la direction que prend la conversation, la détermination du lexique et de la syntaxe constituent des éléments importants de ce choix. De plus, selon Grosjean (1982), divers facteurs peuvent entraîner un changement d'attitude, positif ou négatif, à l'égard d'une langue: reconnaissance officielle d'une langue par le gouvernement, augmentation de l'autonomie de la population, campagne pour les droits civiques, recherches des linguistes et des spécialistes des sciences humaines. L'âge, le sexe, les variantes régionales et dialectales ainsi que le statut intellectuel et social 
sont des facteurs supplémentaires autour desquels gravite le pourquoi de la communication. Ces facteurs déterminent la forme que revêt la langue (Grosjean 1982).

En d'autres termes, la langue, et par extension la communication qu'elle encadre, est inhérente à toute réalité sociale (Anderson 1993).Ainsi, qu'ils soient clairement identifiés ou tenus pour acquis, les signes auditifs et les règles du discours déterminent l'étendue d'une conversation significative. La conversation est donc, comme le rappelle Ingram (1992), le squelette sur lequel sont accrochés les vêtements du discours.

Si la conversation remplit cette fonction, importe-t-il vraiment de savoir comment sont choisis ces «vêtements»? Ne s'agit-il pas simplement d'un choix personnel qu'on met en pratique, qu'on utilise? L'étude de la communication entre les êtres humains apporte de solides arguments propres à favoriser une meilleure compréhension de la façon dont les rapports entre les entendants et les Sourds a évolué et ne cesse d'évoluer.

Dans un tel contexte, la communauté de personnes sourdes a pu maintenir sa propre langue, malgré les nombreux obstacles liés au simple fait d'être minoritaire dans une population composée majoritairement d'entendants. Jetons un regard historique pour évoquer la genèse de certains des problèmes qui persistent aujourd'hui encore.

Nombre de recherches ont déjà indiqué qu'avant le dixhuitième siècle, on qualifiait les Sourds de déficients mentaux (Lane 1984; Padden et Humphries 1988; Woodward 1982). On croyait que la parole dénotait l'intelligence et définissait l'être humain et que seules les personnes capables de s'exprimer oralement étaient en mesure d'enseigner, d'atteindre un statut social élevé, et même de se marier. On peut facilement imaginer la souffrance des personnes sourdes suite à traitement aussi préjudiciable qu'inhumain.

Ce mauvais traitement ne s'est pas dissipé instantanément. Les recherches de Lane montrent en effet que l'abbé de l'Épée et l'abbé Sicard, vers 1760, ont fait œuvre de pionniers en prouvant que les Sourds sont des personnes intelligentes, capables 
d'apprendre une langue et de l'utiliser comme moyen d'instruction. Certes, à cette époque, les apprentissages des personnes sourdes servaient à remplir les poches de leurs enseignants qui s'enorgueillaient, dit-on, de «faire parler les personnes sourdes». Ce faisant, une voie nouvelle, l'éducation, s'ouvrait aux étudiants sourds et c'est avec courage qu'ils ont saisi l'occasion de revendiquer leur droit à la satisfaction de ce besoin humain qu'est la communication (Lane 1984).

En plus de ce stigmate, les Sourds ont dû, au fil des années, surmonter de multiples obstacles. À peine les personnes sourdes entreprenaient-elles l'enseignement en langue des signes, à peine les pensionnats commençaient-ils à se multiplier et à satisfaire aux besoins linguistiques, sociaux et culturels de la communauté sourde, que le décret de Milan fût adopté en 1880. Ce décret interdisait l'emploi de la langue des signes comme moyen pour éduquer les enfants sourds (Padden et Humphries 1988). Heureusement, un groupe américain d'éducateurs de Sourds, sous la direction de Robert P. McGregor, s'est refusé à appliquer un tel décret. Ils ont continué d'inclure la langue des signes dans leur programme d'études, allant à l'encontre du décret selon lequel la seule méthode appropriée d'apprentissage devait être l'«oralisme» ${ }^{1}$ (Gannon 1981).

Dans une optique culturelle, les Sourds ne sont ni victimes d'une maladie, ni le produit d'une déviation génétique. Et pourtant, les scientifiques s'appuyant sur une doctrine pathologique ont souvent cherché à prévenir ou à guérir la surdité. Si, en apparence, leurs buts semblent louables, les recherches de Lane (1993) proposent une nouvelle direction et mettent en garde contre ces scientifiques bien-pensants mais malavisés qui veulent faire disparaître la surdité par la stérilisation, ou encore contre ceux qui font des expériences médicales envahissantes sur les enfants en exécutant une opération chirurgicale de l'implant cochléaire. Il est vrai que les Sourds ont peut-être contracté une maladie ayant entraîné la surdité, mais dans une perspective culturelle, ils sont en pleine possession de leurs moyens. Différents certes, mais non déficients, les Sourds appartiennent à une minorité culturelle. 
Les problèmes liés à la surdité et les jugements péjoratifs sont considérables, mais les personnes sourdes ont dû surmonter une bien plus grande difficulté sur le plan de la communication, parfois même au sein de leur propre famille. En effet, la plupart des enfants sourds grandissent dans une famille d'entendants, car seulement $10 \%$ de la population est sourde. C'est donc dire que le langage des Sourds aurait pu disparaitre en raison de ce facteur d'isolement (Sacks 1990). Heureusement, ce ne fut pas le cas.

Si certains Sourds décident d'accéder au monde des entendants par tous les moyens (le dispositif auditif, l'opération, la méthode orale), d'autres cultivent et s'approprient la langue des signes (LSQ, langue des signes québécoise ou ASL, langue des signes américaine) (Padden 1988). En fait, les personnes sourdes ne désirent pas nécessairement devenir entendantes et n'ont pas forcément besoin de l'être pour se considérer «normales». Du point de vue linguistique et culturel, il pourrait sembler tout à fait naturel que les parents sourds désirent avoir et élever des enfants sourds auxquels ils transmettraient leur héritage. Au lieu d'avoir recours à un implant ou à des appareils auditifs, l'utilisation de la langue des signes et l'interaction avec des adultes sourds constitueraient le meilleur programme d'intervention pour les enfants sourds. D'après mon expérience comme travailleuse sociale, même si les Sourds ont leur propre système de valeurs et de normes, ils se définissent comme «Sourds» et non comme «déficients» (Bienvenu 1991).

Les associations pour les personnes sourdes sont à la fois source de communication, de socialisation et de rencontre pour ceux qui s'intéressent au mariage, et lieu d'échange et d'information sur les nombreuses exigences de la vie. Les anecdotes racontées dans le but de se divertir accompagnent l'histoire des Sourds. De plus, l'art et les objets fabriqués assurent la continuité de leur langue et de leur culture, bref de l'essence même de la communauté sourde.

La force de l'appartenance prévaut sur tout autre moyen quant au choix du type de communication. Tout compte fait, c'est la perception que nous avons de nous-mêmes qui se traduit dans la langue de notre vie. Tel que l'ont souligné de nombreux auteurs, les Sourds sont des individus qui, comme bien d'autres, 
appartiennent à une minorité linguistique et culturelle (Lucas 1989; Padden et Humphries 1988; Woodward 1982). Celui qui ne se sent pas bien dans sa peau risque d'être en proie aux pratiques oppressives et malavisées des autres. Voilà sans doute un des plus importants liens qui se manifeste entre les Sourds et les entendants quand ils cherchent à réconcilier leurs différences linguistiques et culturelles.

Il y aura toujours des gens prêts à dénoncer ceux qui ne sont pas de la même race, de la même religion ou qui ne partagent pas le même point de vue. Mon espoir, c'est que les personnes sourdes continueront à définir et à déclarer leur identité, de revendiquer leurs droits et de trouver ce dont elles ont besoin pour vivre pleinement leur vie. Ce n'est qu'à ce moment que le modèle d'incapacité deviendra un modèle de capacité.

Dans un monde qui privilégie l'individualisme et l'indépendance, il importe de reconnaître la valeur de l'appartenance. Il se peut que cette valeur pousse certains à se fondre dans la foule majoritaire, mais elle en incite d'autres à déclarer ouvertement leur position. Bien que cela puisse sembler un lieu commun, la langue est un facteur déterminant de notre identité et de la façon dont les autres nous perçoivent. Sans avoir aucune caractéristique physique, la langue nous marque tous (K. Rust, conversation personnelle, 1993).

À l'heure actuelle, le défi réside dans le paradoxe suivant: tout en valorisant les différences, on éprouve toujours un certain besoin de se trouver des points communs. Devant un tel dilemme, il se peut bien que nous n'atteignions nos buts que le jour où nos gestes "parleront». Chaque personne possède un droit inné à la communication, droit partagé entre tous, que nous soyons Sourds ou entendants. C'est pourquoi, je conserve l'espoir que les personnes sourdes continueront à s'affirmer, à exiger ce qui leur est dû et à s'ouvrir pleinement à la vie. Ce n'est qu'à ce moment que s'érigera enfin un pont entre le monde des Sourds et des entendants. 


\section{Bibliographie}

ANDERSON,J. (1993) Deaf Student Mis-Writing, Teacher Mis-Reading: English Education and the Deaf College Student, Burtonsville, MD: Linstok Press.

BIENVENU, M.J. (1991). "Can deaf people survive "deafness"?». Perspectives in Deafness, vol. 41, nos 1 et $2,21-25$.

BOWLBY, J. (1973). Attachment and Loss. New York, Basic Books Inc.

FASCOLD, R.(dans Valli, C. et C. Lucas (1992). Linquistics of American Sign Language, Gallaudet University Press.

GANNON, J. (1981)._Deaf Heritage: A Narrative History of Deaf America, National Association of the Deaf.

GROSJEAN, F. (1982). Life with Two Languages, Harvard University Press.

INGRAM, J. (1992). Talk, talk, talk, Toronto, Penguin Books.

KARLEN, N. (1989). «Louder than Words», Rolling Stone, March 23, 133-137.

LANE, H. (1992). The mask of benevolence: Disabling the deaf community, New York, Vintage Books.

LANE, H. (1984). When the Mind Hears: A History of the Deaf, Random House.

PADDEN, C. ET T. HUMPHRIES (1988). Deaf in America:Voices from a Culture, Harvard University Press.

LUCAS, C. (Ed.) (1989). The sociolinquistics of the deaf community, San Dieg, Academic.

SACKS, O. (1990). Seeing voices: A journey into the world of the deaf, New York, Harper Collins.

WATZLAWICK, P., J.H. BEAVIN ET D.D. JACKSON (1967). Pragmatics of human communication, New York, Norton.

WILCOX, S. ET P.WILCOX (1991). Learning to see:American Sign-Language, Englewood Cliffs, NJ, Regents/Prentice Hall.

WOODWARD, J. (1982). How You Gonna Get to Heaven if You Can't Talk with Jesus: The Educational Establishment vs the Deaf Community. Silver Spring, MD, T.J. Publishers, Inc.

WOODWARD, J. (1972). «Implications for sociolinguistics research among the deaf». Sign Language Studies, no 1, 1-7.

\section{Note}

1. L'approche oraliste préconise la seule utilisation de l'oral comme mode de communication en excluant toute forme de gestes. On apprend donc aux Sourds à produire les sons correspondant aux mots en calquant le mouvement labial. 